\title{
Review of microbiological sampling in diabetic foot disease
}

\author{
HANNAH C TRAVERS, JONATHON DAWSON, ANITHA MUTHUSAMI, MICHAEL L WALL
}

\begin{abstract}
Introduction: Diabetes mellitus is a significant cause of morbidity and mortality. Foot-related complications affect $2-2.5 \%$ of people with diabetes. There is significant variation in outcomes for patients with diabetic foot disease within the UK. The multidisciplinary approach to diabetic foot disease is well publicised and protocols, guidance and consensus approaches exist for most components of the management of diabetic foot disease. Antimicrobial therapy to treat diabetic foot infections based on microbiological sampling and culture is well documented, but no consensus exists on how these samples should be obtained, processed and reported. Methods: A literature review was undertaken to establish the reporting of techniques used in obtaining and processing microbiological samples in diabetic foot disease to establish if consensus exists in the methodologies used with a view to develop best practice guidelines.

Results: Six out of 102 papers reported all processes in obtaining and processing microbiological samples.

Conclusion: No gold standard consensus exists for microbiological sampling of diabetic foot infections, preventing optimisation of this aspect of management of diabetic foot disease and ultimately potentially adversely affecting the outcomes of this growing patient cohort.
\end{abstract}

Br J Diabetes 2021;21:233-236

Key words: diabetic foot; microbiology sampling; osteomyelitis

\section{Introduction}

Diabetes mellitus is a significant cause of morbidity and mortality. ${ }^{1}$ Foot-related complications affect $2-2.5 \%$ of people with diabetes, equating to a point prevalence of approximately 58,000 people in England alone. ${ }^{2}$

There is significant regional variation in outcomes for patients with diabetic foot disease within the UK. ${ }^{3}$ The National Diabetes Foot Care Audit aims to quantify these variations at an organisational level so that markers of an effective service can be identified.

Black Country Vascular Network, Dudley Group of Hospitals NHS Foundation Trust, West Midlands.

Address for correspondence: Miss Hannah C Travers Black Country Vascular Network, Dudley Group of Hospitals NHS Foundation Trust, Pensnett Road, Dudley, West Midlands, DY1 2HQ E-mail: hannah.travers@doctors.org.uk

https://doi.org/10.15277/bjd.2021.310
However, low levels of participation have so far made it difficult to draw any consensus on this. ${ }^{4}$

The management of diabetic foot disease is complex, involving input from a multidisciplinary team of professionals. ${ }^{5}$ The mainstays of treatment in these challenging cases are off-loading of pressure areas and appropriate footwear, surgical debridement of infected and necrotic tissue, revascularisation if required, appropriate wound care and dressings, and antimicrobial therapy. Healthcare institutions managing diabetic foot disease should have clear pathways and guidance for management of these patients with alignment of services and processes to ensure the best patient outcomes and reduce major limb amputation rates and the associated morbidity and mortality. ${ }^{4}$

Each facet of the management of diabetic foot disease has been subject to review in the medical literature with consensus documents produced advising on the best practice for the treatment. The use of antimicrobial therapy and prolonged courses to treat osteomyelitis is well documented ${ }^{6}$ and should be based on tissue or bone sampling, culture and appropriate sensitivity testing cultures. ${ }^{5}$ However, how these samples should be obtained, processed and reported is poorly documented, making alignment of services difficult. Targeted antimicrobial therapy relies on certain steps to be completed, as demonstrated in Figure 1. Each of the steps shown has the potential to affect the subsequent accuracy of results and must be clearly described so accurate comparison can be made between techniques and results.

A literature review was undertaken to clarify the practice for reporting of tissue sampling techniques in the diabetic foot population and to determine if consensus exists in the literature for sampling techniques and processing, with the aim of developing best practice guidelines particularly in relation to the intraoperative bone sampling techniques used.

\section{Methods}

The NICE Healthcare Databases Advanced Search (https://hdas.nice.org.uk) was used to search EMBASE and Medline databases in September 2020. The search strategy is detailed in Appendix 1. Studies were restricted to human subjects, in the English language, published between 2010 and 2020 with an abstract available.

A total of 707 papers were identified. Duplicates, case reports and conference abstracts were removed and abstracts were screened by HT and JD for relevance and any conflicts were resolved by the senior author (MW). One hundred and forty-nine full-text articles were deemed relevant for review and 102 were included in the analysis. Figure 2 shows the PRISMA flow diagram. 
Figure 1. The journey of the bone/deep tissue specimen

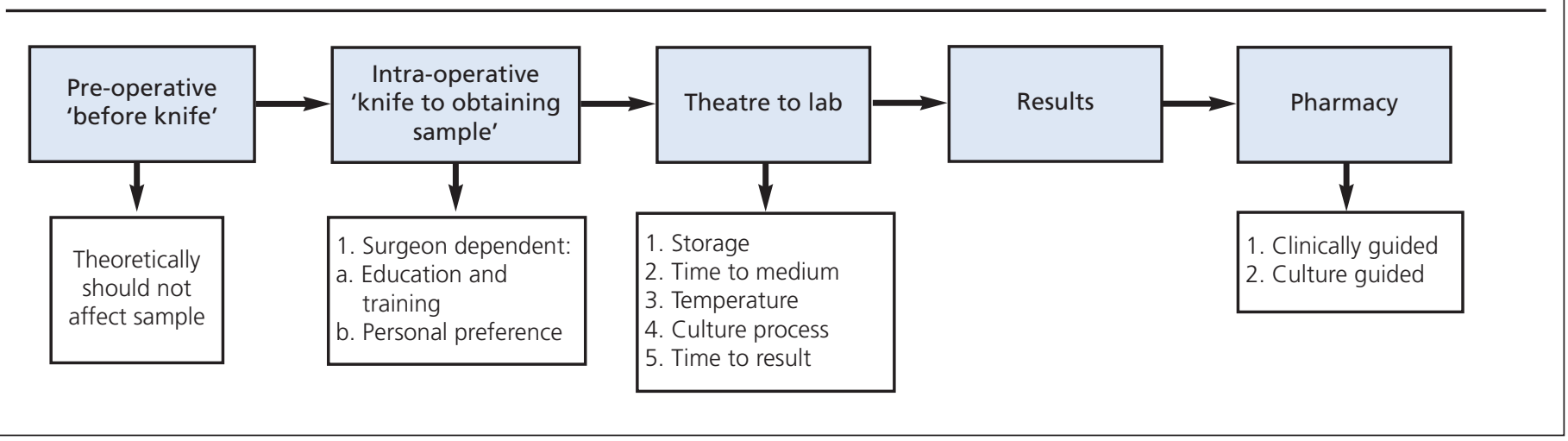

Figure 2. Prisma Flow Diagram

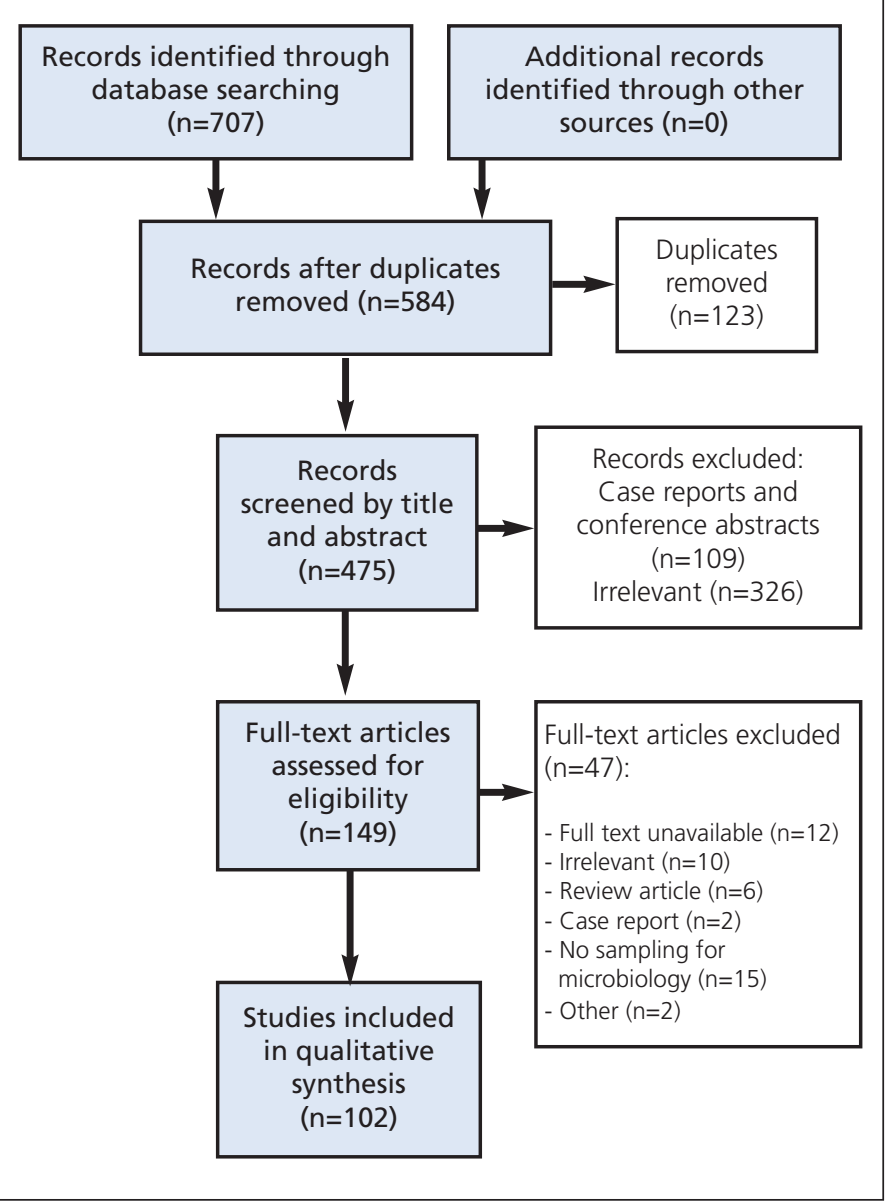

Studies were reviewed by the authors and basic information was collected on the study type and population. The papers were reviewed for the following aspects of their methodology with a view to whether the study would be reproducible: what was sampled, how it was sampled, whether the wound was cleaned prior to sampling and how, how the specimen was transported for processing and what processing occurred. This information was compiled and analysed using Microsoft Excel (Windows 10).

\section{Results}

Of the 707 papers identified through database searching, 123 duplicates and 109 case reports and conference abstracts were removed; 475 were screened by title and abstract and 326 were deemed irrelevant and excluded. Of the 149 full-text articles assessed for eligibility, a further 47 were excluded (reasons detailed in Figure 2). One hundred and two papers were included in qualitative analysis (see Appendix 2), of which 45 were prospective studies, 25 were retrospective studies and in 32 the time frame was unclear. There were 16 observational studies, 1 case series, 3 casecontrol studies, 55 cohort studies, 22 cross-sectional studies, 4 randomised controlled trials and 1 pilot study.

Eighty $(78 \%)$ studies described the sampling technique used, $58(57 \%)$ described how the wound was cleaned prior to sampling, $50(49 \%)$ described how the specimen was kept prior to processing and 80 (78\%) described the processing techniques used.

\section{Samples taken}

Wound or ulcer swabs only were performed in 26 of the papers and pus cultures in five. Bone sampling alone was used in 17 papers, tissue including skin in 17 and other samples in one paper. Thirty-one papers described more than one specimen type being taken.

\section{Sampling technique}

The percentage of papers reporting the use of different techniques for obtaining samples in the systematic review is shown in Table 1.

\section{Wound cleaning}

The percentage of papers reporting how the wound was cleaned prior to microbiology sampling is shown in Table 2.

\section{Specimen transport}

All three variables (time, medium, temperature) of transportation of specimens were reported in $6.9 \%$ of papers, $50 \%$ of papers detailed no information about how the specimen was kept or transported prior to processing, $22.5 \%$ of papers reported only one of the three transport variables (medium 15.7\%, time frame $4.9 \%$, temperature $2.0 \%$ ) and $19 \%$ reported on two of the 
Table 1. Percentage of papers reporting the use of different techniques for obtaining samples in the systematic review

\begin{tabular}{|c|c|c|}
\hline Sample Type & Technique & $\begin{array}{l}\text { Percentage of } \\
\text { papers reporting }\end{array}$ \\
\hline \multirow[t]{3}{*}{ Wound swab } & Levine's & $8.8 \%$ \\
\hline & Other & $5.9 \%$ \\
\hline & $\begin{array}{l}\text { Insufficient description/ } \\
\text { no comment }\end{array}$ & $22.5 \%$ \\
\hline \multirow[t]{2}{*}{ Tissue } & Described & $6.9 \%$ \\
\hline & $\begin{array}{l}\text { Insufficient description/ } \\
\text { no comment }\end{array}$ & $14.7 \%$ \\
\hline \multirow[t]{2}{*}{ Bone } & Described & $6.9 \%$ \\
\hline & $\begin{array}{l}\text { Insufficient description/ } \\
\text { no comment }\end{array}$ & $9.8 \%$ \\
\hline \multirow{3}{*}{$\begin{array}{l}\text { Multiple sample } \\
\text { types }\end{array}$} & Technique specified & $1.0 \%$ \\
\hline & $\begin{array}{l}\text { Samples taken using } \\
\text { "established method" } \\
\text { referencing another paper }\end{array}$ & $2.9 \%$ \\
\hline & $\begin{array}{l}\text { Insufficient description/ } \\
\text { no comment }\end{array}$ & $13.7 \%$ \\
\hline $\begin{array}{l}\text { Other samples } \\
\text { (pus/ fluid/ulcer) }\end{array}$ & $\begin{array}{l}\text { Insufficient description/ } \\
\text { no comment }\end{array}$ & $6.9 \%$ \\
\hline
\end{tabular}

three variables. One paper stated that the specimens were transported by "conventional methods".

\section{Specimen processing}

Detailed processing methods were described in $23.5 \%$ of papers, $30.4 \%$ stated "conventional methods" or "culture and sensitivity" were used, $18.6 \%$ made no comment about the processing techniques, $11.8 \%$ were sent for aerobic and anaerobic culture and $3.9 \%$ for aerobic culture only, and $11.8 \%$ of papers described molecular microbiological techniques.

\section{Complete sampling protocols}

Thirty-five papers (34\%) described all four stages of microbiological sampling and processing and six papers (6\%) sampled bone and described all four stages. These papers were all studies in patients with diabetic foot disease. The techniques described in these six papers are summarised in Appendix 3.

\section{Discussion}

Diabetic foot disease is an international pandemic with a large socioeconomic burden on people and healthcare systems worldwide. Attempts to improve the treatment of diabetic foot disease have been ongoing throughout the medical community with identification of trends in microbiology and the best sampling techniques. Duration of antimicrobial therapy is guided by the culture and sensitivity of samples taken from active diabetic foot infections. Positive bone cultures attract a prolonged (6-week) course of antimicrobial therapy. ${ }^{7,8}$ Inappropriate use of antimicrobials is not without its morbidity and therefore accurate culture and sensitivity is imperative to optimise management.
Table 2. Percentage of papers reporting how the wound was cleaned prior to microbiology sampling

\begin{tabular}{ll}
\hline Method of cleaning & $\begin{array}{l}\text { Percentage of papers } \\
\text { reporting }\end{array}$ \\
\hline $\begin{array}{l}\text { No comment } \\
\text { "Asepsis/ Conventional methods" }\end{array}$ & $42.2 \%$ \\
$\begin{array}{l}\text { Cleaning/ Irrigation - } \\
\text { solution specified }\end{array}$ & $22.5 \%$ \\
$\begin{array}{l}\text { Cleaned/ Irrigation - } \\
\text { solution not specified }\end{array}$ & $5.9 \%$ \\
$\begin{array}{l}\text { "Cleaned (solution specified) } \\
\text { and debrided " }\end{array}$ & $7.8 \%$ \\
$\begin{array}{l}\text { "Cleaned (solution not specified) } \\
\text { and debrided " }\end{array}$ & $3.9 \%$ \\
$\begin{array}{l}\text { Debridement } \\
\text { Multiple steps, well described }\end{array}$ & $6.9 \%$ \\
\end{tabular}

The management of diabetic foot infection requires a multidisciplinary approach and it is the links between specialities that improve patient care. The authors, as surgeons, were concerned that the process by which specimens are sampled and transported to the laboratory for microbiological processing may well be impacting upon the reliability of results. Having standard operating procedures and protocols is well documented in healthcare to improve outcomes; however, there is no gold standard for microbiology sampling and processing to guide antimicrobial therapy in the management of diabetic foot disease. A standardised approach to the sampling process will reduce variation in technique and may help avoid inaccurate results, therefore leading to greater reliability and reproducibility.

There are some limitations to this study. It is a qualitative literature review rather than a systematic review due to the fact that the authors are examining methodology and reporting rather than study results. Non-English language studies were excluded and 12 studies were not available as full-text articles. This may have led to exemplary studies being excluded from this literature review but, if they are not readily available to clinicians treating diabetic foot disease internationally, it is difficult for their results to influence practice.

This literature review clearly demonstrates that there is no standardised methodology for reporting of specimen type, sampling method or processing methods for microbiological culture for the diagnosis and treatment of diabetic foot infection in the medical literature. This heterogeneous reporting means that it is difficult for readers and practitioners to draw accurate conclusions from the published literature in order to improve their own practice or to train the future generation of the multidisciplinary team managing this disease. A recent survey conducted by the author showed a lack of consistency in the sampling techniques in the trainee surgical community. ${ }^{9}$ It also demonstrated a lack of understanding of the processing techniques, procedural reporting and a lack of ongoing training in the surgical debridement of diabetic foot disease, specifically toe amputations.

The authors feel that a consensus must be sought for the sam- 


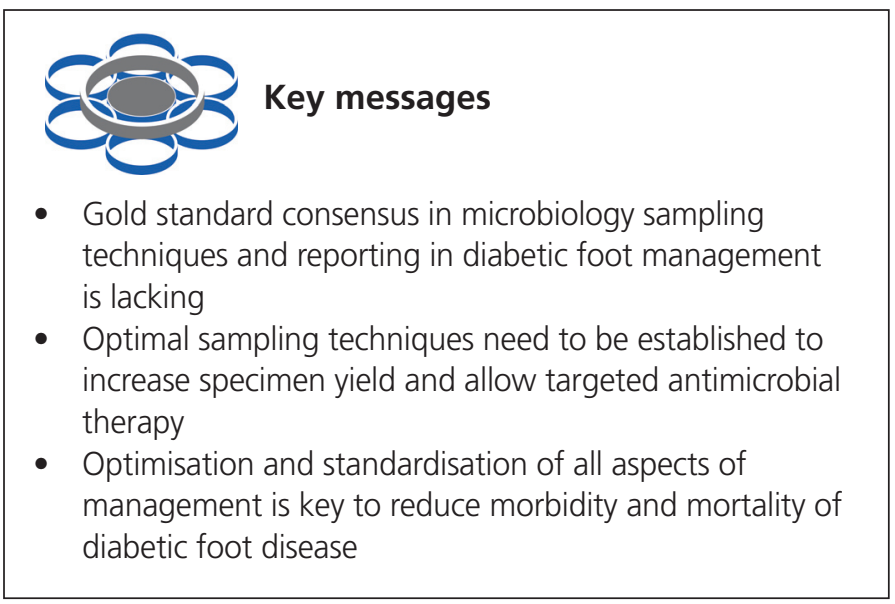

pling and processing of diabetic foot samples. The publication of papers in relation to microbiology sampling in diabetic foot disease must clearly delineate the steps in sampling, transportation and processing, making the studies transparent and reproducible. This will allow the reader to interpret the results and optimise all aspects of management of diabetic foot disease, allow for further studies into techniques, allow rationalisation of antimicrobial therapy and ultimately reduce the long-term sequelae, morbidity and mortality of diabetic foot disease.

Conflict of interest All authors have non to declare. Funding None.

\section{References}

1. Cho NH, Shaw JE, Karuranga S, et al. IDF Diabetes Atlas: Global estimates of diabetes prevalence for 2017 and projections for 2045. Diabetes Res Clin Pract 2018;138:271-81. https://doi.org/10.1016/j.diabres.2018.02.023

2. Kerr M. The cost of diabetic foot disease in England. Diabetic Foot J 2019;22(4):5-6.

3. Jeffcoate $W$, Barron $E$, Lomas J. Using data to tackle the burden of amputation in diabetes. Lancet 2017;390(10105):e29-e30. https://doi.org/10.1016/S0140-6736(17)32401-7

4. Jeffcoate W, Askey A, Berry A, et al. Do we know how good we all are at managing diabetic foot ulcers? A question for those who do not yet participate in the National Diabetes Foot Care Audit. Diabetic Foot $J$ 2020;23(3):8-9.

5. National Institute for Health and Care Excellence. Diabetic foot problems: prevention and management. [NICE Guideline 19]. 2019. Available at: https://www.nice.org.uk/guidance/ng19 (accessed 10 March 2021).

6. Lipsky BA, Berendt AR, Cornia PB, et al. Infectious Diseases Society of America clinical practice guideline for the diagnosis and treatment of diabetic foot infections. J Am Podiatr Med Assoc 2013;103(1):2-7. https://doi.org/10.7547/1030002

7. Tone A, Nguyen S, Devemy F, et al. Six-week versus twelve-week antibiotic therapy for nonsurgically treated diabetic foot osteomyelitis: a multicenter open-label controlled randomized study. Diabetes Care 2015;38(2):302-7. https://doi.org/10.2337/dc14-1514

8. National Institute for Health and Care Excellence. Diabetic foot infection: antimicrobial prescribing. Evidence review. 2019. Available at: https://www.nice.org.uk/guidance/ng19/evidence/evidencereview-pdf-6953995118 (accessed 10 March 2021)

9. Dawson J, Travers HC, Wall ML. Level of training in microbiological sampling for toe amputations in diabetic foot disease: a survey of UK vascular trainees. Diabetic Foot J 2021 (accepted for publication). 
Appendix 1. Search strategy

\section{Search Search Term}

\begin{tabular}{|c|c|}
\hline 1 & exp DIABETES MELLITUS/ \\
\hline 2 & (Diabet*).ti,ab \\
\hline 3 & 1 or 2 \\
\hline 4 & FOOT DISEASES/ \\
\hline 5 & ULCER/ \\
\hline 6 & GANGRENE/ \\
\hline 7 & OSTEOMYELITIS/ \\
\hline 9 & "SOFT TISSUE INFECTION"/ OR "wound infections/" \\
\hline 10 & $(($ foot* OR feet* OR toe* OR tissue* OR wound*) ADJ4 (infect* OR disease*)).ti,ab \\
\hline$\overline{11}$ & (4 OR 5 OR 6 OR 7 OR 9 OR 10) \\
\hline 12 & (3 AND 11) \\
\hline 13 & (diabetic foot).ti,ab \\
\hline 14 & (diabet* ADJ4 (foot* OR feet* OR toe* OR ulcer* OR gangrene* OR osteomyelit*)).ti,ab \\
\hline$\overline{15}$ & (12 OR 13 OR 14) \\
\hline 16 & $($ micro*).ti,ab \\
\hline 17 & (culture).ti,ab \\
\hline 18 & (organis*).ti,ab \\
\hline 19 & $($ sampl*).ti,ab \\
\hline 20 & (16 OR 17 OR 18) \\
\hline 21 & (20 ADJ4 samp*).ti,ab \\
\hline 22 & (19 AND 20) \\
\hline 23 & (21 OR 22) \\
\hline 24 & (15 AND 23) \\
\hline 25 & (15 AND 23) [English language] [Humans] \\
\hline
\end{tabular}


Appendix 2. All papers included in the qualitative review

Year Authors

2020 Macdonald KE et al

2010 Nagoba BS et al

2019 Thanganadar AS et al

2019 Niazi NS et al

2020 Manas AB et al \begin{tabular}{lll}
\hline 2011 & Landsman A et al & $\begin{array}{l}\text { An open-label, three-arm pilot study of the safety and efficacy of topical } \\
\text { levofloxacin versus combined therapy for mild diabetic foot infections }\end{array}$ \\
\hline 2019 Malone $M$ et al & Analysis of proximal bone margins in diabetic foot osteomyelitis by conv
\end{tabular}

Title

A retrospective analysis of the microbiology of diabetic foot infections at a Scottish tertiary hospital A simple and effective approach for the treatment of diabetic foot ulcers with different Wagner grades A Study on isolation, characterization, and exploration of multiantibiotic-resistant bacteria in the wound site of diabetic foot ulcer patients Adjuvant antibiotic loaded bio composite in the management of diabetic foot osteomyelitis - a multicentre study Admission time deep swab specimens compared with surgical bone sampling in hospitalized individuals with diabetic foot osteomyelitis and soft tissue infection Analysis of proximal bone margins in diabetic foot osteomyelitis by conventional culture, DNA sequencing and microscopy

2016 Wolcott RD et al Analysis of the chronic wound microbiota of 2,963 patients by 16S rDNA pyrosequencing

2020 Monami M et al Antimicrobial photodynamic therapy in infected diabetic foot ulcers: a multicenter preliminary experience

2018 Pugazhendhi S and Dorairaj AP Appraisal of biofilm formation in diabetic foot infections by comparing phenotypic methods with the ultrastructural analysis

2019 Lavery LA et al Are we misdiagnosing diabetic foot osteomyelitis? Is the gold standard gold?

2020 Min KR et al

2018 Vatan A et al

2016 Karmaker $\mathrm{M}$ et al

2017 Sanchez-Sanchez M et al

2020 Ullah I et al

2017 Amjad SS et al

2018 Yasin $\mathrm{M}$ et al

2010 Sotto $A$ et al

2015 Lipsky BA et al

2014 Murali TS et al

2020 Goh TC et al

2012 Mendes JJ et al

2018 Kim PJ et al

2011 Zubair M et al

2018 Nelson A et al

2016 Nelson EA et al

2019 Bellazreg F et al

2011 Lesens $\mathrm{O}$ et al

2013 Aslangul E et al

2012 Sotto $A$ et al

2018 Wu M et al

2017 Malone $\mathrm{M}$ et al

2019 Malone $\mathrm{M}$ et al

2018 Saseedharan $\mathrm{S}$ et al

2016 Reveles KR et al

2019 MacDonald A et al

2019 Couturier $\mathrm{A}$ et al
Association between baseline abundance of Peptoniphilus, a Gram-positive anaerobic coccus, and wound healing outcomes of DFUs

Association between biofilm and multi/extensive drug resistance in diabetic foot infection Association of bacteria in diabetic and non-diabetic foot infection - an investigation in patients from Bangladesh Bacterial prevalence and antibiotic resistance in clinical isolates of diabetic foot ulcers in the Northeast of Tamaulipas, Mexico

Bacteriological profile and antibiotic susceptibility patterns In diabetic foot infections at Lady Reading Hospital, Peshawar Bacteriology of diabetic foot in tertiary care hospital; frequency, antibiotic susceptibility and risk factors Baseline characteristics of infected foot ulcers in patients with diabetes at a tertiary care hospital in Pakistan Beneficial effects of implementing guidelines on microbiology and costs of infected diabetic foot ulcers Ceftaroline fosamil for treatment of diabetic foot infections: the CAPTURE study experience. Characteristics of microbial drug resistance and its correlates in chronic diabetic foot ulcer infections.

Clinical and bacteriological profile of diabetic foot infections in a tertiary care

Clinical and bacteriological survey of diabetic foot infections in Lisbon

Clinic-based debridement of chronic ulcers has minimal impact on bacteria

Clinico-microbiological study and antimicrobial drug resistance profile of diabetic foot infections in North India

CODIFI (Concordance in Diabetic Foot Ulcer Infection): a cross-sectional study of wound swab versus tissue sampling in infected diabetic foot ulcers in England

Concordance in diabetic foot ulceration: A cross-sectional study of agreement between wound swabbing and tissue sampling in infected ulcers

Correlation between superficial and intra-operative specimens in diabetic foot infections: Results of a crosssectional Tunisian study

Culture of per-wound bone specimens: A simplified approach for the medical management of diabetic foot osteomyelitis

Diagnosing diabetic foot osteomyelitis in patients without signs of soft tissue infection by coupling hybrid 67Ga SPECT/CT with bedside percutaneous bone puncture.

Distinguishing colonization from infection with Staphylococcus aureus in diabetic foot ulcers with miniaturized oligonucleotide arrays: a French multicenter study

Distribution of microbes and drug susceptibility in patients with diabetic foot infections in Southwest China

Effect of Cadexomer iodine on the microbial load and diversity of chronic non-healing diabetic foot ulcers complicated by biofilm in vivo

Effect on total microbial load and community composition with two vs six-week topical Cadexomer iodine for treating chronic biofilm infections in diabetic foot ulcers

Epidemiology of diabetic foot infections in a reference tertiary hospital in India

Epidemiology of methicillin-resistant Staphylococcus aureus diabetic foot infections in a large academic hospital: implications for antimicrobial stewardship

Evidence of differential microbiomes in healing versus non-healing diabetic foot ulcers prior to and following foot salvage therapy

Comparison of microbiological results obtained from per-wound bone biopsies versus transcutaneous bone biopsies in diabetic foot osteomyelitis: a prospective cohort study

continued... 
Year Authors

2018 Elmarsafi T et al

2017 Esposito S et al

2013 Malone $\mathrm{M}$ et al

2011 Tascini $C$ et al

2018 Noor S et al

2013 Djahmi N et al

2017 Oli AN et al

2016 Smith K et al

2014 Mannucci E et al
Title

Concordance between bone pathology and bone culture for the diagnosis of osteomyelitis in the presence of Charcot neuro-osteoarthropathy

Deep tissue biopsy vs. superficial swab culture, including microbial loading determination, in the microbiological assessment of skin and soft tissue infections (SSTIs)

Deep wound cultures correlate well with bone biopsy culture in diabetic foot osteomyelitis

Microbiology at first visit of moderate-to-severe diabetic foot infection with antimicrobial activity and a survey of quinolone monotherapy

Molecular and culture based assessment of bacterial pathogens in subjects with diabetic foot ulcer

Molecular epidemiology of staphylococcus aureus strains isolated from inpatients with infected diabetic foot ulcers in an Algerian University Hospital

Multi-antibiotic resistant extended-spectrum beta-lactamase producing bacteria pose a challenge to the effective treatment of wound and skin infections

One step closer to understanding the role of bacteria in diabetic foot ulcers: Characterising the microbiome of ulcers

Photodynamic topical antimicrobial therapy for infected foot ulcers in patients with diabetes: A randomized double-blind, placebo-controlled study - The D.A.N.T.E (Diabetic ulcer Antimicrobial New Topical treatment Evaluation) study

2010 Saltoglu $\mathrm{N}$ et al $\quad$ Piperacillin/tazobactam versus imipenem/cilastatin for severe diabetic foot infections: A prospective, randomized clinical trial in a university hospital

2015 DaCosta RS et al Point-of-care autofluorescence imaging for real-time sampling and treatment guidance of bioburden in chronic wounds: first-in-human results

2014 Dunyach-Remy $\mathrm{C}$ et al Polymerase chain reaction-denaturing gradient gel electrophoresis (PCR-DGGE): A promising tool to diagnose bacterial infections in diabetic foot ulcers

2011 Bernard L et al Predicting the pathogen of diabetic toe osteomyelitis by two consecutive ulcer cultures with bone contact

2017 Chisman R et al

2019 Jaju K et al

2014 Merlet A et al

2013 Redel $\mathrm{H}$ et al

2012 Atway $S$ et al

$2011 \quad$ Elamurugan TP et al

2019 Sloan TJ et al

2018 Jneid J et al

2019 Beroukhim G et al

2020 Kosmopoulou OA et al

2013 Aragon-Sanchez J et al

2011 Weiner RD et al

2016 Kumar D et al Prescribing antibiotics in diabetic foot infection: what is the role of initial microscopy and culture of tissue samples? Profile and antibiotic susceptibility of bacterial pathogens associated with diabetic foot ulcers from a rural area Prognostic factors of calcaneal osteomyelitis Quantitation and composition of cutaneous microbiota in diabetic and nondiabetic men

Rate of residual osteomyelitis after partial foot amputation in diabetic patients: a standardized method for evaluating bone margins with intraoperative culture.

Role of bone biopsy specimen culture in the management of diabetic foot osteomyelitis

Examining diabetic heel ulcers through an ecological lens: Microbial community dynamics associated with healing and infection

Exploring the microbiota of diabetic foot infections with culturomics

Factors predicting positive culture in CT-guided bone biopsy performed for suspected osteomyelitis

Feasibility of percutaneous bone biopsy as part of the management of diabetic foot osteomyelitis in a 100\%

neuropathic, grade 3 IDSA/IWGDF population on an outpatient basis

Gram-negative diabetic foot osteomyelitis: Risk factors and clinical presentation

Histology versus microbiology for accuracy in identification of osteomyelitis in the diabetic foot Identification, antifungal resistance profile, in vitro biofilm formation and ultrastructural characteristics of Candida species isolated from diabetic foot patients in Northern India

2017 Ottolino-Perry K et al Improved detection of clinically relevant wound bacteria using autofluorescence image-guided sampling in diabetic foot ulcers

2013 Ray GT et al Incidence, microbiology, and patient characteristics of skin and soft-tissue infections in a U.S. population: a retrospective population-based study.

Increasing incidence of Gram-negative organisms in bacterial agents isolated from diabetic foot ulcers

2013 Turhan V et al

2015 Cervantes-García E et al

2017 Noor S et al

2019 Park J et al

infections of diabetic foot ulcers with methicillin-resistant Staphylococcus aureus

Inflammatory markers as risk factors for infection with multidrug-resistant microbes in diabetic foot subjects

Influence of microbiota on diabetic foot wound in comparison with adjacent normal skin based on the clinical features

2018 Saltoglu $\mathrm{N}$ et al

2014 Boffeli TJ et al Influence of multidrug resistant organisms on the outcome of diabetic foot infection

In-office distal Symes lesser toe amputation: a safe, reliable, and cost-effective treatment of diabetes-related tip of toe ulcers complicated by osteomyelitis

2018 Makki D et al Is it necessary to change instruments between sampling sites when taking multiple tissue specimens in musculoskeletal infections?

2011 Vinodkumar CS et al Isolation of bacteriophages to multi-drug resistant Enterococci obtained from diabetic foot: a novel antimicrobial agent waiting in the shelf?

2018 Meyr AJ et al Level of agreement with a multi-test approach to the diagnosis of diabetic foot osteomyelitis

2017 Dunyach-Remy C et al Link between nasal carriage of Staphylococcus aureus and infected diabetic foot ulcers 
Year Authors

2018 Ramanujam CL et al

2018 Suryaletha K et al

2012 Parvez $\mathrm{N}$ et al

2013 Islam S et al

2020 Pontes DG et al

2012 Tiwari $S$ et al

2015 Parsa $\mathrm{H}$ et al

2017 Miyan Z et al

2014 Sugandhi P et al

2018 Shettigar K et al

2018 Drampalos E et al

2017 Kassam NA et al

2016 Fujii $M$ et al

2018 Chang JW et al

2013 Malik A et al

2020 Crisologo PA et al

2019 Banerjee T et al

2016 Nageen $A$

2013 Gardner SE et al

2012 Abbas Z et al

2020 Hunter $P$ et al

2012 Pinzur MS et al

2019 Johani $\mathrm{K}$ et al

2016 Shettigar K et al

2018 Haalboom M et al
Title

Medical imaging and laboratory analysis of diagnostic accuracy in 107 consecutive hospitalized patients with diabetic foot osteomyelitis and partial foot amputations

Metataxonomic approach to decipher the polymicrobial burden in diabetic foot ulcer and its biofilm mode of infection

Microbial profile and utility of soft tissue, pus, and bone cultures in diagnosing diabetic foot infections

Microbial profile of diabetic foot infections in Trinidad and Tobago

Microbiologic characteristics and antibiotic resistance rates of diabetic foot infections

Microbiological and clinical characteristics of diabetic foot infections in northern India.

Microbiological features and risk factors in patients with diabetic foot ulcers

Microbiological pattern of diabetic foot infections at a tertiary care center in a developing country

Microbiological profile of bacterial pathogens from diabetic foot infections in tertiary care hospitals, Salem

Severity of drug resistance and co-existence of Enterococcus faecalis in diabetic foot ulcer infections

Single stage treatment of diabetic calcaneal osteomyelitis with an absorbable gentamicin-loaded calcium sulphate/hydroxyapatite biocomposite: The Silo technique

Spectrum and antibiogram of bacteria isolated from patients presenting with infected wounds in a tertiary hospital, northern Tanzania.

Surgical treatment strategy for diabetic forefoot osteomyelitis

The appropriate management algorithm for diabetic foot: A single-center retrospective study over 12 years

The diabetic foot infections: Biofilms and antimicrobial resistance

The infected diabetic foot: Can serum biomarkers predict osteomyelitis after hospital discharge for diabetic foot infections?

The microbiology of diabetic foot infections in patients recently treated with antibiotic therapy: A prospective study from India

The microflora of chronic diabetic foot ulcers based on culture and molecular examination: a descriptive study The most prevalent organism in diabetic foot ulcers and its drug sensitivity and resistance to different standard antibiotics

The neuropathic diabetic foot ulcer microbiome is associated with clinical factors

The utility of Gram stains and culture in the management of limb ulcers in persons with diabetes

Topical oxygen therapy shifts microbiome dynamics in chronic diabetic foot ulcers

Treatment of osteomyelitis in charcot foot with single-stage resection of infection, correction of deformity, and maintenance with ring fixation

Understanding the microbiome of diabetic foot osteomyelitis: insights from molecular and microscopic approaches

Virulence determinants in clinical Staphylococcus aureus from monomicrobial and polymicrobial infections of diabetic foot ulcers

Wound swab and wound biopsy yield similar culture results 
Appendix 3. Summary of papers reporting all aspects of bone sampling techniques and processing in diabetic foot patients

\begin{tabular}{|c|c|c|c|c|c|c|}
\hline & 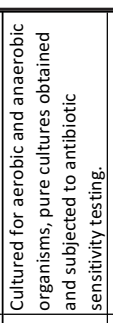 & 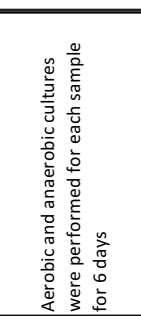 & 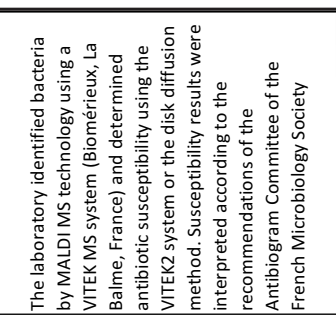 & 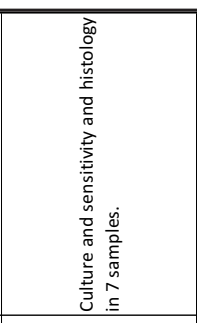 & 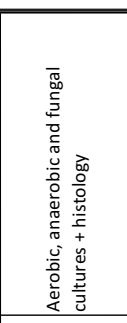 & 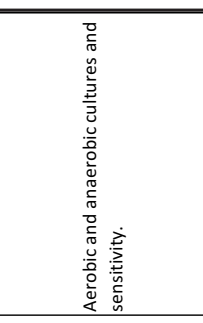 \\
\hline & 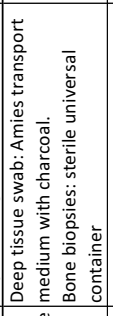 & 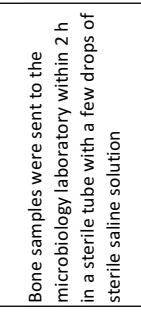 & 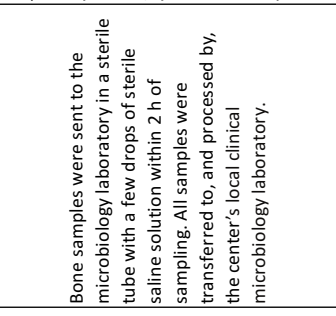 & 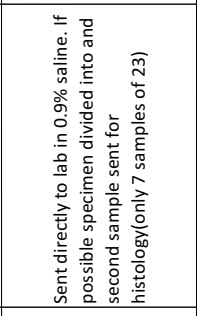 & 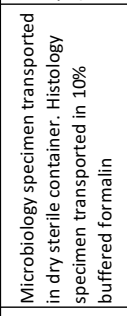 & 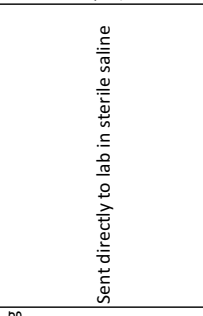 \\
\hline 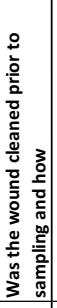 & 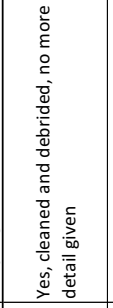 & 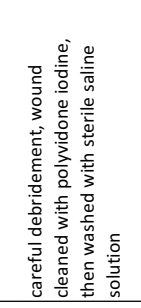 & 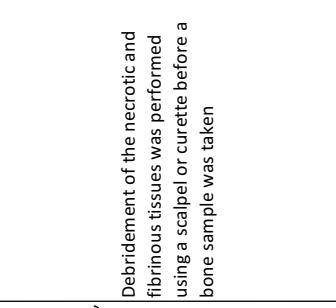 & 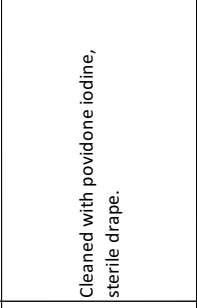 & 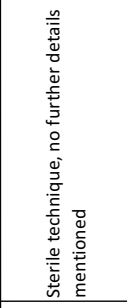 & 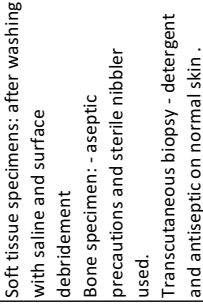 \\
\hline 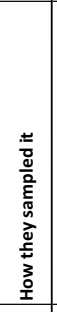 & 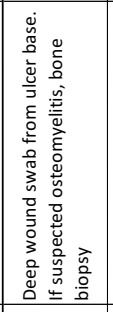 & 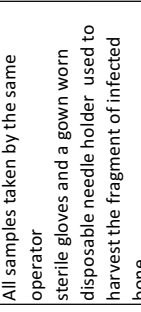 & 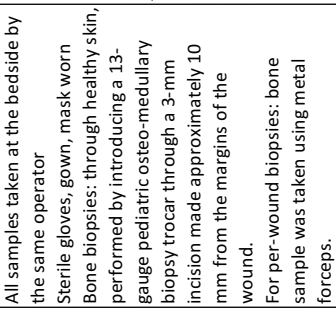 & 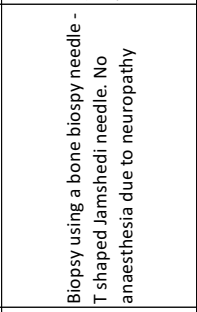 & 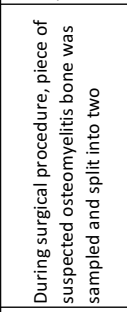 & 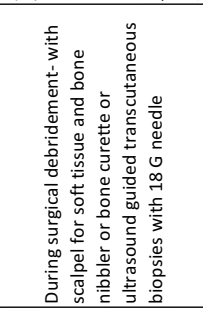 \\
\hline 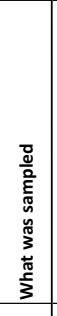 & 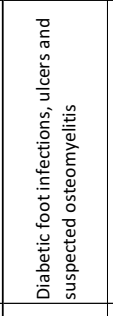 & 总 & 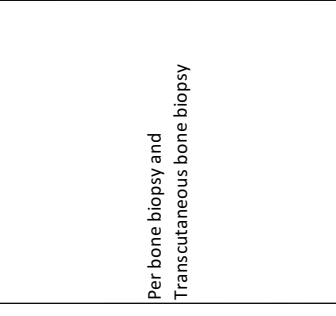 & 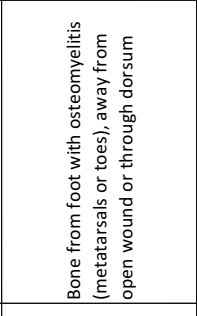 & 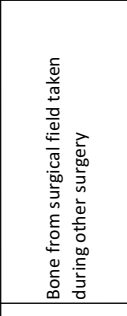 & 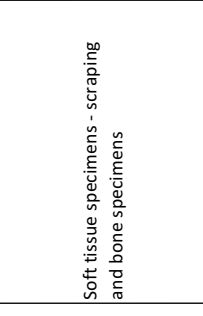 \\
\hline 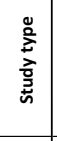 & 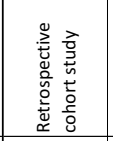 & 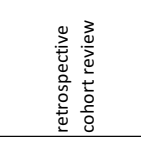 & & 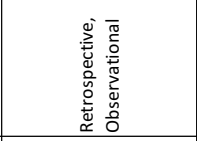 & 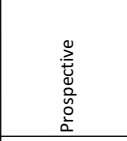 & 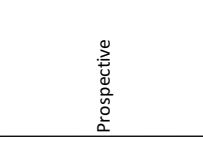 \\
\hline 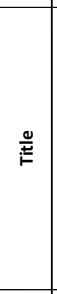 & 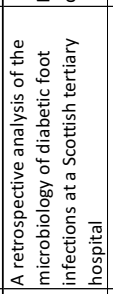 & 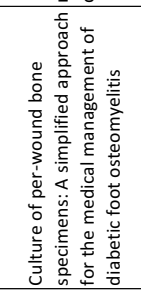 & 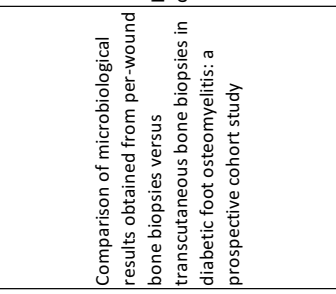 & 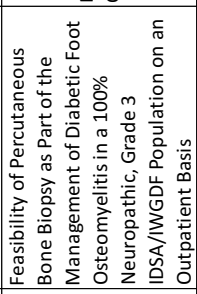 & 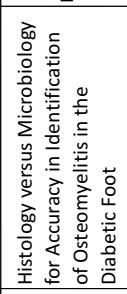 & 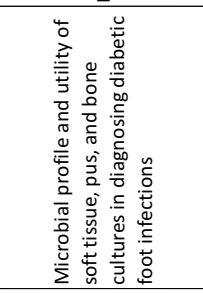 \\
\hline 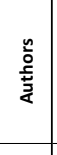 & 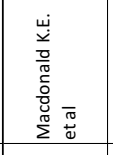 & 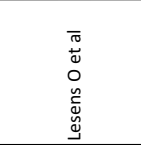 & 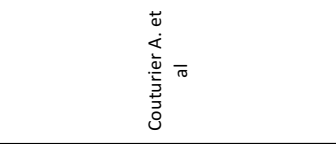 & 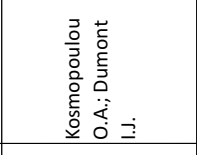 & 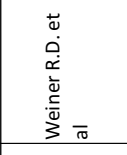 & 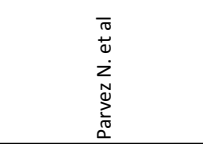 \\
\hline 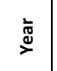 & జి & ت્ & 亏े & ปี & $\overrightarrow{\tilde{N}}$ & సี \\
\hline
\end{tabular}

